\title{
Cyberdating: Misinformation and (Dis)trust in Online Interaction
}

\author{
Hong Wang and Xin-An Lu \\ Department of Human Communication Studies \\ Shippensburg University of Pennsylvania \\ Shippensburg, PA, USA
}

howang@ship.edu; xalu@ship.edu

\begin{abstract}
Through an analysis of the unique features of the Internet as an environment for cyberdating, this work examines the issues of (mis)information and (dis)trust in romantic endeavors and encounters in the virtual world. With a critical application of Grice's Cooperative Principle to a reported case of fraud in cyberdating, this paper offers practical suggestions for cyberdaters.
\end{abstract}

Keywords: cyberdating, media analysis, misinformation, trust, cooperative principle, interpretation of electronic messages.

\section{Introduction}

With the exponential boom and prevalence of the Internet, virtual space has become an alternative and additional venue for romantic ventures. "Cyberdating," as it is called, promises to proffer new possibilities and creativities not found in the real-life world. The multi-billion dollar industry in Internet dating and matching services attests to the popularity of cyberdating. This popularity may be attributable to the Internet's flexible accessibility, creative opportunities, and perhaps most importantly, anonymity and new freedoms attending that anonymity. These freedoms remove and disentangle netizens from the moral and social qualms in the face-to-face world and facilitate a "free play" with identity and imagination.

However, with the new possibilities of cyberdating come new problems characteristic of this new arena for romance. Misinformation, for instance, has been proved a troubling phenomenon in cyberdating, whose dangers and treacherous nature have been plentifully covered in the popular press. Take a quick a look at the following titles: "When spouses go astray online"; "Man with over 200 online wives"; "Internet romance ends with man jailed in Wales"; and "French woman dies after an Internet romance sours". More dramatic tragedies in cyberdating involve kidnapping, abuse, and even murder. Therefore, of great practicality are studies of the unique features of the

Material published as part of this publication, either on-line or in print, is copyrighted by the Informing Science Institute. Permission to make digital or paper copy of part or all of these works for personal or classroom use is granted without fee provided that the copies are not made or distributed for profit or commercial advantage AND that copies 1) bear this notice in full and 2) give the full citation on the first page. It is permissible to abstract these works so long as credit is given. To copy in all other cases or to republish or to post on a server or to redistribute to lists requires specific permission and payment of a fee. Contact Publisher@InformingScience.org to request redistribution permission. medium of the Internet, which engender and implicate new issues and problems for human interaction in the mediated space.

This paper begins with an analysis of the media characteristics of cyber space that tend to render information more malleable and the incidence of misinformation more likely. Second, examination will be given of the dual nature of online interaction: misinformation at the 
Sender's end and (dis)trust at the Receiver's end. Third, this work investigates how trust is gained and lost in cyberdating. What principles and behaviors affect the operation of trust in cyberdating? Do online encounters commence with assumed trust or must trust be acquired in the process of deepened interactions? With the citation of a reported case of fraud in cyberdating, the fourth section of this work illustrates and analyzes the process of misinformation detection. The paper ends with practical implications for cyberdaters and their relational development.

\section{The Internet as a Unique Medium for Dating}

Based on the fundamental premise of McLuhan's (1964) postulate that "the medium is the message", this section discusses the media characteristics of the Internet. Specifically, the following factors of the Internet as a medium will be examined: immediacy of feedback, multiple cues, language variety, and personal focus. This examination reveals that the media characteristics of the Internet facilitate easier manipulation of information and increase the incidence of misinformation.

\section{Medium as the Message}

When a new technology emerges, its form merits careful study, for "the medium is the message", as admonished by McLuhan (1964). A medium exerts influence on the society by not only playing a role in its delivered content, but by way of the very mechanism of its media characteristics. McLuhan illustrated this point with the invention of the light bulb. A light bulb does not possess or deliver content in the way of a newspaper article or television program. It has no content, but it creates space. The space created by the light bulb offers new possibilities; for instance, it enables people to work in what otherwise would be complete darkness. In this sense, a light bulb, by its mere presence, creates a new environment or medium, with new possibilities and new influences (McLuhan, 1964, p. 8). Similarly, the invention of the Internet does not merely give a new tool for information collection and circulation; it creates a virtual world that awakens and develops new dimensions of human interactions, revises the sense of authenticity, and excites new imaginations. Research finds that different communication media, for the social purpose of uncertainty reduction, vary in their abilities to convey knowledge and information (Daft \& Lengel, 1984, 1987; Newberry, 2001; Trevino, Lengel, \& Daft,, 1987). A medium, such as face-to-face communication or electronic mail, may be labeled "rich" or "lean" depending on the following four abilities: (1) immediacy of feedback; (2) capacity to transmit multiple cues; (3) language variety; and (4) personal focus (Daft \& Lengel, 1984). What follows is a discussion of these four capabilities of the Internet and their implications for cyberdating. For extended discussion of media "richness", readers may also consult Dennis and Valacich (1999), who added parallelism, rehearsability and reprocessability to the list.

\section{Immediacy of Feedback}

"Immediacy of feedback" refers to a medium's speed in transmitting feedback. A "richer" medium provides more immediate feedback, making it easier for communicators to adjust their responses to received messages (such as signaling understanding, confusion, or disagreement).

Some electronic communication channels such as the E-mail, Instant Messenger (IM), Multiple User Dialogue (MUD) enable cyberdaters to receive much faster replies from their partners than with the traditional (postal) mail. However, compared with face-to-face communication, these electronic media may have a more limited capability for feedback immediacy, as electronic communication is not as mutually immediate as face-to-face communication. The bulk of electronic communication still happens largely in two separate steps: message sending and then message receiving. Synchronization in face-to-face communication may appear more pronounced, even compared with the so-called synchronous electronic communication of video chatting and video 
conference. The face-to-face conversationalist does not have to finish, for instance, "I lo(ve)" before the partner can chime in.

Communication studies suggest that interpersonal relationship development depends heavily on instant and simultaneous interactions. Even more important is the individual's ability to freely and simultaneously switch between the roles of Sender and Receiver. Thus, face-to-face interaction seems to provide more feedback immediacy and better facilitates relational growth than does electronic communication. The Internet's inferior feedback immediacy may cause a certain degree of frustration and thus negatively impact communicators' attitude toward information quality and trust acquisition in cyberdating.

\section{Multiple Cues}

"Multiple cues" mean the medium's ability to convey varied types of information that appeal to different senses of the communicators. Rich media allow a fuller range of verbal, paralinguistic, intonation, proxemic, kinetic, olfactory, and tactile cues to convey the subtleties and implications of a message in addition to the message's literal content (Lim \& Benbasat, 2000). Some researchers call the "surplus" cues of rich media "social cues" as they enhance social interaction (Daft, Lengel, \& Trevino, 1987; Farmer \& Hyatt, 1994). The Internet may deprive cyberdaters of numerous "social cues" that are available in face-to-face communication (e.g., the tactile and the olfactory). Both research and intuitive experience inform that such factors as gender, age, personal appearance, and physical build carry great significance in the development of romantic relationships, especially in the early stages. Absent of the desired visual appeals, a relationship will not start or may end abruptly. As the relationship grows, visual, auditory, olfactory, and tactile conveyance of motions and emotions remain strongly desired. Evidently, communicators depend on a large spectrum of signs of various types for "true" information that is necessary for trust acquisition and maintenance. Suspicion is likely when the communicator, for instance, detects avoidance of eye contact and other discomfort in conversation. In addition, communicators rely on the consistency of behavioral patterns for detection of misinformation or lies. Behavioral consistency is generally associated with honesty and inconsistency with deception regardless of the specific behaviors that are enacted (Henningsen, Cruz, \& Morr, 2000). In its present state and compared with face-to-face communication, the Internet has limited capabilities in providing the full range of social cues that are necessary to facilitate information authentication and trust acquisition.

\section{Language Variety}

"Language variety" relates to the range and scope of meaning that language symbols may convey (Farmer \& Hyatt, 1994). For instance, numbers generally convey more precise meaning than natural language (Daft et al., 1987), and graphic symbols carry a greater range of interpretations (Daft \& Lengel, 1984). Some information may be easier to convey in one format than in another. Facial features are often more accurately portrayed in a photograph, whereas an action is better captured in a video. The Internet may enhance the transmission of quantifiable or "codify-able" information. An online communication about a math problem and its calculation may be less daunting compared with an oral explanation of the same via just face-to-face interaction. Romantic relationships, however, tend to focus on non-quantifiable, difficult-to-code expressions of emotions. Mere textual transcriptions, on which much electronic media rely, contribute to the troubling incidence of unintended implication and interpretation. To help their understanding of an online textual message, cyberdaters often resort to contrived techniques to reduce qualitative emotions to quantifiable codes required by the electronic media. Keyboard characters are used to simulate facial expressions and paralinguistic features of conversation, such creations often coined as "emoticons" or emotional icons (e.g., :-) or (-) to imply a smiling face). The person that 
wishes to have his/her message interpreted as friendly teasing, for example, may have to interpose the extra and extraneous word or phrase of "grin" or "just kidding" (Parks \& Floyd, 1996). The consequence of low language variety of the Internet will receive more discussion in the fourth section of this paper.

\section{Personal Focus}

"Personal focus" refers to a medium's ability to facilitate the communicators' expression of personal feelings and emotions. For greater personal focus, messages need to be tailored to the frame of reference, needs, and current situation of the receiver (Daft et al., 1987). Greater "personal focus" helps to project the communicator socially and affectively into a community of inquiry (Rourke, Anderson, Garrison, \& Archer, 1999; Russo, 2000). A rich medium, such as face-toface interaction, may offer greater personal focus by enabling the interactant to see the partner as a real, physical human being. In cyberdating, however, the participant is one step removed from the partner and addresses but the immediate presence of an inanimate computer, compromising the personal focus of electronic messages.

Discussion of feedback immediacy, multiple cues, language variety, and personal focus helps to reveal the unique media features of the virtual world. These features may represent a doubleedged sword to cyberdaters. On the one hand, electronic communication better serves cyberdaters' need for a greater quantity of information and enables them to multi-communicate with several people at the same time. The anonymity and physical absence of the interactants offer exciting space of imagination and creativity. However, the media "leanness" of the Internet deprives cyberdaters of important social cues that are necessary for information authentication and trust acquisition. Misinformation and distrust thus become compelling issues to confront and plague cyberdaters. The following sections of the paper will address such issues and suggest compensatory methods for cyberdaters to remedy the "leanness" of the electronic medium.

\section{Misinformation: What does it mean?}

Misinformation in cyberdating may be approached from three angles: intention, reception, and situational desirability. The term misinformation is used here to mean the discrepancy between what a word or a sign (or a series of signs) signifies and the reality it references. Misinformation, though generally perceived as negative, may be more prevalent than we are willing to acknowledge. A candid reflection on misinformation, adhearing strictly to the above definition, may point to a near omnipresence of misinformation in everyday life. The prevalence of misinformation may become understandable when we reflect on the great difficulty not to misinform. Any given piece of information simply cannot contain the multifaceted and variegated totality of reality. "A white sheet of paper" fails to inform about the shades of whiteness, the length, width, and thickness of the paper, its quality, desirability, and availability. When asked "How are you?" people more than likely choose to offer the simple response of "doing fine" and will not delve into every annoying detail in their life. "I love you" means, in frank analysis, "I love you at this time and place when I am feeling this emotion." The simple expression of "I love you" may have missed the temporal and spatial frame of reference, as hardly any love could inoculate itself from the corrosive detritus of time, life, and society. Misinformation is so deep-seated in everyday life that much of it is unconsciously treated as innocuous. However, misinformation in cyberdating could be much less innocuous.

Framed against the triad of intention, reception, and situational desirability, this section of the paper addresses how misinformation is interpreted and perceived. Discussion will also be offered on how cyberdaters interpret and perceive misinformation. 


\section{Misinformation and Intention}

If interpreted according to the definition discussed earlier, misinformation could hardly be avoided in any normal conversation. Popular interpretation of misinformation, however, often associates it with intention. For distinction, some scholars choose to label unintended inaccurate information as "disinformation." A piece of information will only be interpreted and perceived as misinformation when a strategic purpose is assigned to the distortion of this information. The mundane and oft-used term of "lying" refers to what our more scholarly term of "misinformation" purports to denote here. According to Reboul (2001), "The speaker of a lie has the intention to produce a specific effect: that his hearer should believe something which the speaker takes, rightly or wrongly, to be false" (p. 20). Grice (1989) identifies two major ways to achieve the intended effect of a lie: (1) You can say something you believe to be false and get your hearer to perceive it as true. (2) You can say something you believe to be true and get your hearer to take it as false. Much misinformation in cyberdating tends to revolve around personal identity and social status, including one's age, looks, height, profession, and income, all of which may seem important for meaningful relational development. Next, the paper will discuss the reception and detection of misinformation and how situational desirability affects the interpretation of misinformation.

\section{Misinformation and Reception}

From the Receiver's perspective, misinformation will not be so unless it is discovered and recognized as such by the Receiver. Absence of cognizance of misinformation signifies true information to the Receiver. For instance, a man may remain a perfect date, humorous, well-off, and kind, until the woman finds out that he is already married with three kids. The revelation brings the woman to realize what she'd thought to be true information is in fact misinformation. Such a discovery requires that the Receiver relies on the following data: (1) the conventional meaning of the words used and the references signified by these words; (2) rules that both the Sender and Receiver observe; (3) the context of the utterance; (4) items of background knowledge; and (5) the fact claimed or implied by the previous four factors is available for verification by both Sender and Receiver (Grice, 1989, p. 30). These five types of necessary data for detection of misinformation, or rather the difficulty involved in their acquisition, make misinformation detection a rather arduous task in cyberdating. Data types four and five may be especially so for cyberdaters.

\section{Misinformation and Situational Desirability}

Different cyberdaters may manifest different reactions and attitudes toward the same piece of (mis)information, depending on their individual expectations for cyberdating. For some, the desirable outcome may be to find the true love with whom to "live happily ever after". For this group, authentic information is crucial. Disappointment is immediate upon the detection of falsehood and misinformation, especially when it involves such important factors as age, physical build, gender, and economic status. Hardey (2004) corroborates that informational authenticity is valued in many cyberdating sites where successful communication depends on the development of trust between strangers.

However, discovery of the "true love" may not be the end result desired by other cyberdaters. These cyberdaters dive into the virtual space perhaps to find the new, the mysterious, the alternative, and the excitement that are not readily available in the face-to-face world. Textual exchange and the virtual world created thereby may prove already adequate for these cyberdaters' expectations and their platonic wish for "emotional communication, with others and with the self" (Giddens, 1992, p. 130). Such cyberdaters may be contented, for instance, if the exchanged messages resonate with their deep feelings and emotional vibrations; whether the messages originate from a puppy, a young girl, or an old man may be of little concern. Investigation and verification of in- 
formation authenticity may be of much less importance to this group as opposed to the previous group of "seekers of the true love". Deviations from mundane reality and hackneyed truths (such as creative identities and imagined locations of an ethereal nature) may even be what these cyberdaters strive to achieve.

When cyberspace is intended as an initial step that will hopefully extend into face-to-face interactions, misinformation, nevertheless, must be approached seriously. Otherwise, cyberdaters may fall victim to real crimes and physical harm, as depicted by those titles in popular press cited in the introduction of this paper.

For cyberdaters that wish to extend their cyber-interactions into the real world, misinformation and the corollary issue of trust remain significant. As discussed in section one of this paper, the "lean" nature of the Internet makes it difficult to detect misinformation, authenticate true information, and develop trust. However, trust acquisition and development is not impossible in cyberdating. The next section of the paper will discuss principles governing trust, and how these principles may apply to message and emotional exchanges for the "cyber-construction" of trust.

\section{Trust: Where does it come from?}

Do cyberdaters commence their romantic ventures with an assumed trust before proved wrong? Or do they start with a suspicious distrust until actual trust is obtained? According to Grice (1989), trust operates on two levels: (1) trust of cooperative intention between the two parties; and (2) acceptance of the exchanged message content as true. To illustrate, John asks if Mary would join him in an online chat. Mary says that she noticed a worm that eats up everything. John may feel hurt because Mary's response implies she is not paying attention to his invitation. Mary does not manifest a "cooperative intention" by meaningfully responding to John's question. John's trust in Mary for a bona fide online chat may in turn be lost. However, pre-established interactions with Mary may lead John to a different interpretation of Mary's message. Mary's response, perceived as one of apathy to a stranger, may mean a solid "yes" to John, as John knows well that is Mary's idiosyncratic manner of saying "yes".

The very presence of the cyberdater in virtual space may communicate the trust of cooperative intention, a purposeful intention to participate in online dating. However, lack of pre-established and pre-online interaction with the partner complicates Grice's second level of trust in cyberdating. The following section of the paper introduces Grice's maxims of cooperative principle that govern the operation of trust and discusses how this principle may apply to cyberdating and affect trust therein.

\section{Grice's Cooperative Principle}

In 1975 Grice proposed that there is an accepted way of speaking, endorsed by all participants as standard behavior. In other words, meaningful execution of communication presupposes a communal agreement on certain behavioral principles. Grice calls this agreement the Cooperative Principle (CP). He further articulates $\mathrm{CP}$ into four major maxims:

1. The maxim of quality:

Try to make your contribution one that either you believe or have evidence to verify its trueness.

(a) do not say what you believe to be false

(b) do not say what you can not prove 
2.The maxim of quantity:

Make your contribution as informative as is required; no more or no less for the current purpose of the exchange.

3. The maxim of relation:

Be relevant to the topic of the current exchange

4. The maxim of manner:

Be clear in your intention and concise in your expression

According to the cooperative principle, when people send or receive an utterance in purposeful communication, they assume that the utterance is true, provides adequate information, and is relevant and dependable. However, practice in real life communication testifies both to the evident and widespread belief in the social norm of cooperative principle, and to the frequent breach of this norm. As a result, two distinct responses are equally possible toward infraction of the cooperative principle. One, it is perceived as violation of the cooperative principle and commission of falsehood, leading to loss of trust. Two, it is perceived to insinuate an alternative or unusual connotation, or in Grice's term, conversational implicature. For illustration, let's use the following simple conversational transaction:

Tom: How's your new date?

Tracy: A big success.

Tom: Oh, Ok (with either a wry or sympathetic smile).

For Tom, Tracy's response is evidently her attempt to address his question, therefore, relevant. Tracy answers his question in no ambiguous terms and thus observes the maxim of manner (or clarity). In addition, Tracy seems to be honoring the maxim of quantity, not saying more or less than required by Tom's question. However, in terms of the maxim of quality (or truthfulness), Tom may derive two different interpretations of Tracy's response. One, Tracy is telling the truth as her date is actually a success and a great satisfaction to her. Two, Tracy's new date turns out to be a total disaster; Tracy's message either attempts to dissemble the truth or is intended as irony and she wants no more discussion of her dating nightmare. Both of Tom's interpretations could prove plausible in real communication. Tom's interpretation of Mary's response as truth or falsehood, as direct response or irony, or as observance or breach of the maxim of quality, may largely need to depend on the way that Mary utters the response. Extra-textual cues are of indispensable service here, corroborating the popular saying that the real message may lie in the unsaid. "Hearers assume that an utterance addressed to them is intended to be meaningful. Therefore, if the utterance doesn't have an appropriate conventional meaning, they will look for a more useful interpretation" (Davies, 2000, p. 18). It is frequently the availability of extra-textual cues that help generate alternative interpretations of ambiguous messages and in turn aid the Receiver to grasp the real and implied intention (or the conversational implicature) of the Sender.

Among Grice's four maxims of cooperative principle - quality, quantity, relevance, and manner - the last three are relatively easy to determine. Textual content and context may enable the Receiver to arrive at a rather quick verdict on whether these three maxims are observed. However, the maxim of quality proves to be more problematic. The policing and determination of the observance of the maxim of quality in cyberdating is elusive, to say the least. As illustrated by the above simple conversation between Tom and Tracy, textual transactions alone are inadequate for an accurate verdict over the observance of the maxim of quality. A much larger range of communicational or social cues need to be exploited to grasp conversational implicatures. The Internet's lack of such cues and its elusive social accountability make problematic the determination of 
whether the maxim of quality is honored. What follows is a discussion of this issue and of how trust operates in cyberdating.

\section{Cooperative Principle and Trust in Cyberdating}

In cyber romance, as in any other romantic interaction, the first level of trust (i.e., cooperative intention) is distinctively there. The very presence of the cyberdater in the virtual space communicates a purposeful intention to participate and cooperate in electronic communication. However, the second level of trust - trust in truthfulness - is another issue. Clear manifestation of the first level of trust, compounded with the elusive nature of the second level of trust, makes cyberdating a rather intriguing and yet often dilemmatic experience. The cyberdater may be confounded by the disparity between the unequivocal trust of cooperative intention and the suspicion over textual messages. The "leanness" of the Internet as a medium simply exacerbates the problem.

Trust of content, different from trust of the cooperative intention, cannot be assumed but has to be actually acquired. The Internet's limited capabilities in providing multiple cues spur cyberdaters to make adaptations of traditional means to acquire the second level of trust. Many, especially those engaged in textual chatting, choose to focus less on the actual looks and other physical aspects of the partner, and more on internal qualities of the partner (e.g., educational level and social finesse as derived from textual messages). The more lax observance of social norms in cyberspace may actually help a quick revelation of one's true state in nonphysical aspects.

However, studies (e.g., Merkle \& Richardson, 2000) have shown that physical attractiveness and attitudinal similarity are important factors to develop an inchoate encounter into a sustained relationship. Physical attractiveness in initial romantic encounters seems to clearly outweigh other factors. A minute detail in physical appearance may lead to either a hasty intensification of interest or an abrupt termination of interest in a stranger.

Lack of visual and certainly tactile and olfactory elements in the majority of online dating channels favors a propensity toward the nonphysical and spiritual qualities of a partner. Physical attractiveness may give way to personality, as constructed and presented by the partner's textually coded thoughts. If governed by the cooperative principle, the nimbly synchronized maneuvers between reading, writing and message volleying may efficiently guide the cyberdater to a rather accurate gauge of the partner's personality, values, and attitudes. Shared "vocabularies of bodily idioms", viewed by Goffman (1959) as central to the "presentation of self" in public, are exchanged in the cyberspace for the development of cyber-relationships, through which a (virtual) sense of trust is constructed.

Grice's maxim of manner or clarity is often compromised in face-to-face interactions because of social etiquette. Although clarity and efficiency in communication may call for blunt terms, people typically opt to pleasant terms, particularly in expression of negative feelings at the early stage of relational development. Under the pressure of social decency, self-disclosure may come late and progress at a pace endorsed by relational development. A communicator could be easily offended, for instance, if a stranger abruptly initiates the question, "Hello, how old are you?" or if the stranger discloses too many personal secrets too early. However, one step removed and more liberated from the restraint of social etiquette, cyberdaters seem to self-disclose much more often and much more quickly. Upon immediate arrival into the chatroom, the cyberdater may fire a barrage of questions for efficient collection of important information about his/her the partner (e.g., age, sex, physical features).

Merkle and Richardson (2000) speculated that this change of norms and expectations is due to the anonymity provided by the Internet, greatly reducing participants' social qualms. Reduced visual and auditory cues may also play a role in facilitating self-disclosure. However, the laxity of social etiquette in cyberspace could prove both a boon and a bane. On the one hand, communication 
could be liberated from social restraints so as to focus more on clarity, efficiency, and hard truths. On the other hand, the same features of the Internet may slacken participants' sense of caution, facilitates information manipulation and dissemblance, and contribute to the occurrence of fraud and misinformation. Other factors may also play into the operation of trust in cyberdating: less spatial confinement, greater difficulty to physically track the participant, elusive behavioral accountability, and lack of relational sustainability due to easy and flippant initiations and terminations of interactions. More studies may prove necessary to investigate the negative and positive effects that the distinctive medium features of the Internet could exert on the operation of trust in cyberdating. By citing one case of cyberdating, the following section empirically delineates how trust in the cyberspace is assumed, acted upon, and then lost when misinformation is detected.

\section{An Example of Misinformation in Cyberdating}

Initiation and continuation of meaningful communication in the cyberspace still requires observance of the cooperative principle. Lack of such observance, when detected, may lead to an immediate termination of communication. The problem is how to effectively detect online breach of the cooperative principle. The lack of multiple cues and the absence of pre-established or preonline interactions fail to establish a background that is essential for authentication of information, detection of misinformation, and construction of trust. Conversational implicatures in cyberspace become much more elusive. Cyberdaters that observe the cooperative principle fall easy prey for those that do not. This section of the paper depicts a case of cyberdating to illustrate how online trust could be assumed, acted upon, abused, and then lost. This section will also discuss how the detection of online fraud often requires extension of the online interaction into the faceto-face world, bringing the cyberdater into the tangible danger inherent in the physical world.

\section{A Narrated Case of Cyberdating and Fraud}

This case is cited from www.wenxuecity.com ("Man with over 200 online wives," 2003), a major Chinese website featuring news, entertainment, and forums. The story is narrated in first person allegedly by a young lady. She accused a man of online cheating by exploiting the trust of more than 200 of his "virtual wives":

I came across D-Cat, the man, in a private chat room. After initial greeting and selfintroduction, D-Cat offered to tell me that he'd just come back from Germany after four long years. I had learned from a female cyber friend that D-Cat had offered to give her a cellular phone for "further contacts". I was not involved with D-Cat in any romantic sense, and did not plan that way. I was only curious as to why he had made that cellular phone offer to that female friend of mine. He explained that he once owned "emotional debts" to the female gender [the original wording is nü xing-female gender. Contextually, it should be interpreted as that particular cyber friend of the narrator, or the "sweetheart", in D-Cat's term], and wanted to make it up materially. In addition, he was suffering from cancer, so money had lost much of its meaning for him.

The turning point was at our first face-to-face meeting. D-Cat did not make a very positive impression on me with his disproportioned body shape and a big paunch. I also noticed that he has thick hair and healthy complexion that could hardly be associated with chemo patients. At this meeting, he revealed more of himself. His father died when he was little. His stepfather, who was in the steel business, had paid hundreds of thousands of dollars for treatment of the young man's lung cancer. Seeing that I looked sympathetic, he asked me to lend him some money so that he could make calls to his "sweetheart". This rhetoric of money borrowing did not strike me as plausible, plus the fact that he neither looked nor sounded like suffering from a fatal disease. All the clues so far turned my suspicion on. Soon after our face-to-face meeting, I managed to get his password and got 
into his QQ mailbox. To my surprise, he had over 200 female cyber friends, all of whom he referred to as his "dear wives". He abused their trust in him, and cheated them out of their money [when they placed trust in him]...

In this narrated interaction, D-Cat was guilty of patent misinformation, with strategy and premeditation. He lured the other gender with material attractions (e.g., the promise to buy the girl friend a cellular phone); he lied about his background, and particularly his health condition for the strategic purpose of currying others' sympathy; he cheated hundreds of people out of their money. The reader may question the validity of this particular piece of narrative, which could be a perfect fabrication. However, as our focus in this work is analysis of trust in the cyberspace, we might as well overlook the authenticity of the story itself, and focus instead on the "hypothetical realism" of the story to facilitate our analysis. What follows is an analysis of how misinformation is detected and trust lost in the narrated story.

\section{Analysis of Misinformation Detection in the Case}

It is not easy to acquire complete data to determine how well Grice's cooperative principle is observed in a communicational transaction. The difficulty may be exacerbated for cyberdaters as they have to rely on limited channels of lean information in the cyberspace. Authentication of information or detection of misinformation becomes treacherous tasks. Relaxed social norms and lack of behavioral accountability may also contribute to the relative ease of the production of misinformation and deception in cyberspace.

Before her face-to-face meeting with D-Cat, the narrator in the previous story found herself passively interpreting the messages the way they were strategically and deceptively designed. On the surface, every verbal message exchanged fit in with the Cooperative Principle; she did not detect any flouting or breach of the maxims. The only signal of extra-textual meaning (i.e., meaning in addition to that generated by the textual message) arose with the topic of the cellular phone. Her real-life experience led her to believe that material offering in a cyber relationship might signal hidden motives - "young men nowadays are very realistic; they won't spend time and money for you if they could not get sexual gratification and/or more money in return", as she later related. Unfortunately, the discrepancy between what her cyber partner verbally communicated and what her life experience taught her serves as the only indicator of possible breaching of the maxim of quality. She has little further means to gauge her partner's conversational implicature. Her suspicion remains on the level of conjecture without solid verification. Cyberdating hangs its participants frequently in the exciting and troubling air of uncertainties and speculations.

Conversational implicatures began to make sense to the narrator only after her face-to-face encounter with her partner, D-Cat. The added visual cues in the face-to-face encounter enabled her to verify what she read against what she saw, revealing misinformation in her partner's electronic communication regarding his cancer. This revelation alerted her to exercise caution. D-Cat's further communication in the face-to-face encounter corroborated the narrator's suspicion. He began to disclose to her about his father and stepfather, a self-disclosure that went beyond what social norms would endorse at the early stage of relational development. In this sense, D-Cat violated the maxim of quantity, which requires the Speaker to tell no more or no less than warranted by conventional needs of communication. Although Internet users may pardon and accept in cyberspace a higher level of self-disclosure that may appear inappropriate in face-to-face encounters, violation of the maxim of quantity in real-life world certainly raises the red-flag of suspicion. DCat's excessive disclosure in the face-to-face interaction inclined the narrator toward a suspicion of ulterior motives. D-Cat's expressed interest to borrow money clinched the narrator's suspicion. Chinese culture frowns upon an effort to borrow money from strangers (which D-Cat and the narrator were except for their immature electronic and impersonal exchanges in cyberspace). All of such detection, however, came as a result of face-to-face encounter, which provides many com- 
municational cues unavailable in the cyberspace. Sole reliance on cyber-communication may not lead the narrator to the same conclusion.

It is not coincident in the narrated case that verbal messages began to generate new implicatures during the face-to-face interaction. Face-to-face encounters are the desired and indispensable extension of cyberdating for many participants, as the former provides cyberdaters numerous benefits unavailable in the latter. In a comparative study between online and offline romantic relationships, McDowell (2001) listed sixteen types of turning points in relational development. The following are the three types where online relational development becomes statistically significantly different from offline relation: (1) initial face-to-face meeting, (2) significant shifts in media, and (3) exclusivity. McDowell finds that the initial face-to-face meeting with one's significant other (either a positive or negative experience) is considered to be the most important turning point in relational development, leading to crucial changes in commitment levels. The initial face-to-face meeting was mentioned almost exclusively by the online group, ranked as the third most important turning point by this group. For the offline group, however, initial face-to-face encounter appeared as the least important turning point in relational development $\left(16^{\text {th }}\right)$. For other critical turning points, online participants cited "significant shifts in media or in exchange of personal information" 12 times as often as do the offline group. Significant shifts in media refers to the expansion of communication to new types of media (e.g., from phone calls to email exchanges, from email to photo sharing). Significant shifts in exchange of personal information refer to changes in revelation of personal information (e.g., from disclosure of first names to that of last names, from disclosure of real age to exchanging of phone numbers or email addresses). The different reports by the online and offline groups regarding critical turning points in relational development may be because shift in media in online relationships represent significant increases in the number of channels (e.g., vocal, visual). Media changes for cyberdaters often mean a new and otherwise unavailable ability to gain additional information about the partner (McDowell, 2001).

Another research by Parks and Roberts (1998) reports that, of the respondents that initiated relationships on a MOO (Multi-User Dimensions, Object Oriented, a real-time, text-based virtual environment), $80 \%$ had also contacted their partner via email; $66.8 \%$ had spoken on the telephone; $54.5 \%$ had communicated by writing (cards or letters); $40.5 \%$ had exchanged photos by mail; and $37.7 \%$ reported that they had gone on to meet their partner in person. Partners in romantic relationships were the most likely to eventually meet face-to-face $(57.9 \%$ according to McDowell, 2001). This is confirmed by reports of mail, telephone, photo exchange as additional means that supplement computer-mediated communication (Ogan, 1993; Parks \& Floyd, 1996; Reid, 1991).

Discussion and analysis in this section indicate that computer-mediated communication, due to various limitations in communicational capability, may not be a self-sufficient channel of communication for meaningful development and fruition of romantic relationships. Supplementary channels of communication (e.g., phones, traditional mail, face-to-face interactions) prove desirable and even indispensable. The following section points cyberdaters to additional suggestions and reminders for their online romantic ventures.

\section{Implications for Cyberdaters in Information Exchange}

Up to now, we have discussed the medium characteristics unique to the Internet, examined misinformation and its reception and interpretation, and analyzed trust as governed by Grice's cooperative principle and its application in cyberdating. We illustrated the operation of trust and its loss in cyberdating via a cited case of fraud. To bring all the above onto a more practical level for cyberdaters, the final section of this paper offers practical suggestions and reminders implied by the previous discussion. 


\section{Distinction between Two Levels of Trust}

As with offline interaction, cyberdaters need to be aware of the two distinct and yet overlapping levels of trust - that of the cooperative intention and that of message truthfulness. As the former serves as the pre-requisite for the initiation of meaningful interaction, its existence may be assumed in cyberdating. The latter, however, must be worked out and actually achieved via information authentication and/or misinformation detection. As the Internet is a rather lean medium for human interaction, frequently depriving the communicators of valuable and essential communicational cues, cyberdaters need to exercise extra caution regarding the possibility of misinformation. Information authentication and verification must occur before trust is given. Cyberdaters may find it necessary to employ supplementary channels to remedy the "leanness" of electronic interactions. Yet at the same time cyberdaters need to be aware of the potential risks and new complications that may arise when electronic interaction is extended into other modalities of communication (especially face-to-face communication).

\section{Separation between Sender Role and Receiver Role}

Keep in mind that the Sender and the Receiver are not identical roles. Each is associated with distinct assumptions and strategies. This distinction is of special significance in cyberdating. The Receiver needs to trust the sent message so as to create the requisite condition for the initiation of interaction. The Sender in cyberspace, however, is obliged only by the self-policed social etiquette of truthfulness. As the Sender may choose to manipulate and distort information for negative strategic purposes, the Receiver has to go the extra mile to figure out the authenticity and truthfulness of the Sender's information. Success in the real world's face-to-face communication requires competence in both roles and adept switching between the roles that is appropriate to the situation or the participant's wishes. In cyberspace where communication frequently occurs in delayed steps, people need to distinguish the two roles so as to see more clearly what is possible with the role of the Sender and what to do in the role of Receiver.

\section{The Double-Edged Sword of Online Anonymity}

Cyberspace facilitates the accomplishment of anonymity. Anonymity, however, can be both a boon and a bane, creating exciting possibilities and engendering troubling risks. Cyberdaters are often enthusiastic about the new freedoms and imaginations that online anonymity brings to them. In the virtual space, they feel disentangled and emancipated from the sticky web of social norms and moral restraints. They are ready for some "free play" with new identities. They are eager to dive into the deepest truths of human existence. They see cyberspace as a freed new venue for truth, nothing but the blunt truth. However, the new freedoms in cyberspace can also be abused for strategic manipulations that are not endorsed and even condemned in the real world. Online anonymity, for instance, makes it harder, if not impossible, to track physically communication participants and to assure behavioral accountability. Lack of spatial confinement makes the cyberspace a free market. Anyone could come, leave, or express themselves in a manner that does not have to heed even the slightest principle of social decency. Cyberdaters must not forget that the evil edge of the sword of cyberspace may be used just as often as the noble edge.

\section{Risk of Extension of Online Interaction}

Because of the limitations of cyberdating (e.g., lack of tactile and olfactory senses), many participants strive to extend their virtual interaction into physical contact. However, the cyberdater must understand that premises of online interaction greatly differ from those of face-to-face interaction. The former may proceed and continue in the haze of fabricated identities and imagined existences. In other words, the former could proceed with assumed trust or even in the absence of trust. Face-to-face communication, however, need to proceed on the solid foundation of verified 
identities and truthful information. Success of face-to-face communication depends on established trust. Communication in the real world, if based on the gossamer premises of online communication, may lead to disasters of rape and murder. Limitations of cyberspace admonish cyberdaters to realistically frame their expectations for cyberdating. Extension of cyberdating into real dating must be handled with caution and with modification of foundational premises. Cyber participants may choose to be contented with the experience of just "talking" to their cyber soulmates for platonic understanding, care, affection, and comfort. It is advisable to refrain from physical contact before online interactions have produced the mature conditions required for successful communication in the real world.

\section{Conclusion}

An anonymous quote may serve as an apt endnote to conclude this work: "Online dating sites and chat rooms make it easy for predators to find victims, phishing scams trick people into giving away their life savings and children can come across inappropriate material far too easily. Then again, life before the Internet was not devoid of problems. Predators still found victims, chain letters and other forms of scams still managed to swindle people out of their money, and children could still be exposed to filth. Instead of creating these problems, it would seem the Internet is guilty of making it easier for them to happen. It's also possible that the frequency of these problems has not increased, but that the wealth of information we now enjoy makes it appear that the problems are more frequent than before."

This paper argues that the medium characteristics unique to the Internet clearly differentiate cyberdating from dating in the real world. The creation, reception, and interpretation of misinformation functions differently in the virtual space. Trust emerges and operates differently in the virtual space. Premises that enable online interaction may prove immature to produce meaningful faceto-face communication. Cyberdaters must understand the different nature of virtual interaction in order to successfully exploit the Internet's exciting possibilities and wisely avoid undesirable and tragic consequences when online interaction is tactlessly extended into real world face-to-face encounters.

The readers may have noticed that this work largely confines itself to the discussion of the most popular and the more traditional modality for cyberdating - textual chatting. New developments in Internet technology are adding new communicational capabilities to the Internet, such as visuality and even a simulated sense of tactility. Further studies of these new capabilities of the Internet are necessary to further the investigation of human interaction in the virtual space. The nature of discussion in this paper is communicational. However, factors that influence human interaction in the mediated space of the Internet may not be just communicational. They may range from the low speed of the Internet to the manageability of large files, from lack of spatial confinement in the virtual world to lack of the olfactory sense. Non-communicational factors may also merit further investigation in the study of mediated human interaction.

Aided with computer technology and particularly the Internet, mediated human interaction has revolutionarily extended face-to-face interaction into new possibilities and new problems. Traditional issues in face-to-face human interaction such as misinformation and trust, once positioned in the mediated space, seem to acquire different facets and pose new issues. Studies of mediated human interaction promise fertile land of research for scholars of human communication. This paper, in the authors' humble hope, serves as another invitation for exploitation of such fertile land of scholarship. 


\section{References}

Daft, R.L. \& Lengel, R.H. (1984). Information richness: A new approach to managerial behavior and organizational design. In L.L. Cummings \& B.M. Staw (Eds.), Research in organizational behavior, Vol 6, (pp. 191-233). Homewood, IL: JAI Press.

Daft, R.L. \& Lengel, R.H. (1986). Organizational information requirements, media richness and structural design. Management Science, 32(5), 554-571.

Daft, R.L., Lengel, R.H., \& Trevino, L.K. (1987). Message equivocality, media selection, and manager performance: Implications for information systems. MIS Quarterly, 11, 355-366.

Davies, B. (2000). Grice's cooperative principle: Getting the meaning across [electronic version]. In D. Nelson \& P. Foulkes (Eds.), Leeds working papers in linguistics. Retrieved March 9, 2006, from http://www.leeds.ac.uk/linguistics/research/WP2000/Davies.pdf

Dennis, A. \& Valacich, J. (1999). Rethinking media richness: Towards a theory of media synchronicity. In Proceedings of the 32nd Hawaii International Conference on System Sciences. Retrieved April 30, 2006, from http://csdl.computer.org/comp/proceedings/hicss/1999/0001/01/00011017.PDF

Farmer, S. M. \& Hyatt, C. W. (1994). Effects of task language demands and task complexity on computermediated work groups. Small Group Research, 25, 331-366.

Giddens, A. (1992). The transformation of intimacy: Sexuality, love \& eroticism in modern societies. Stanford, CA: Stanford University Press.

Goffman, E. (1959). The Presentation of Self in Everyday Life. Garden City, NY: Doubleday.

Grice, H. P. (1975). Logic and conversation. In P. Cole and J. Morgan (Eds.), Syntax and Semantics (Vol. 3) (pp. 41-58). New York: Academic Press.

Grice, H. P. (1989). Studies in the ways of words. Cambridge, MA: Harvard University Press.

Hardey, M. (2004). Mediated relationships: Authenticity and the possibility of romance. Information, communication and society, 7(2), 207-222. Retrieved March 10, 2006, from http://www.tandf.co.uk/journals

Henningsen, D. D., Cruz, M. G. \& Morr, M. C. (2000). Pattern violations and perceptions of deception. Communication Reports, 13(1), 1-9.

Lim, K. H. \& Benbasat, I. (2000). The effect of multimedia on perceived equivocality and perceived usefulness of information systems. MIS Quarterly 24(3). Retrieved March 24, 2006, from http://www.misq.org/archivist/vol/no24/issue3/vol24n3art4.html

Man with over 200 online wives. Retrieved March 3, 2003 from http://www.wenxuecity.com/BBSview

McDowell, S. W. (2001). The development of online and offline romantic relationships: A turning point study. Retrieved March 3, 2003, from http://www.internetromance.org/thesis4.html

McLuhan, M. (1964). Understanding media: The extensions of man. New York: McGraw-Hill.

Merkle, E. R. \& Richardson, R. A. (2000). Digital dating and virtual relating: Conceptualizing computer mediated romantic relationships. Family Relations, 49, 187-192.

Newberry, B. (2001). Raising student social presence in online classes. In W. Fowler \& J. Hasebrook (Eds.), Proceedings of WebNet 2001 World Conference on the WWW and the Internet (pp. 905-910). Norfolk, VA: Association for the Advancement of Computing in Education (AACE).

Ogan, C. (1993). Listserver communication during the Gulf War: What kind of medium is the electronic bulletin board? Journal of Broadcasting and Electronic Media, 37, 177-196.

Parks, M. R. \& Floyd, K. (1996). Making friends in cyberspace. Journal of Computer-Mediated Communication, 1(4). Retrieved March 3, 2003, from http://jcmc.huji.ac.il/vol1/issue4/vol1no4.html 
Parks, M.R., \& Roberts, L.D. (1998). 'Making MOOsic': The development of personal relationships on line and a comparison to their off-line counterparts. Journal of Social \& Personal Relationships, 15(4), 517-538.

Reboul, A. (2001). Semantic transparency, semantic opacity, states of affairs, mental states and speech acts. In L. Anolli, R. Cieri, \& G. Riva (Eds.), Say not to say: New perspectives on miscommunication. Amsterdam: IOS Press.

Reid, E. (1991). Electropolis: Communication and community on Internet relay chat. Unpublished honors thesis, Department of History, University of Melbourne, Melbourne, Australia.

Rourke, L., Anderson, T., Garrison, D. R., \& Archer, W. (1999). Assessing social presence in asynchronous, text-based computer conferencing. Journal of Distance Education, 14(3) 51-70. Retrieved March 24, 2006, from http://cade.athabascau.ca/vol14.2/rourke et_al.html

Russo, T. (2000, May). Social presence: Teaching and learning with invisible others. Paper presented at Creating Effective Online Instruction, University of Kansas, Lawrence, KS.

Trevino, L.K., Lengel, R.K. \& Daft, R.L. (1987). Media symbolism, media richness and media choice in organizations. Communication Research, 14(5), 553-574.

\section{Biographies}

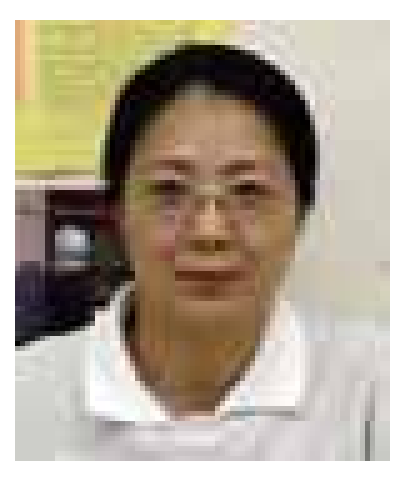

Hong Wang holds a Ph.D. in Speech Communication from Southern Illinois University-Carbondale. She is currently an Assistant Professor in the Department of Human Communication Studies at Shippensburg University of Pennsylvania. She has published numerous articles and book chapters on semiotics, linguistics, and communication studies.

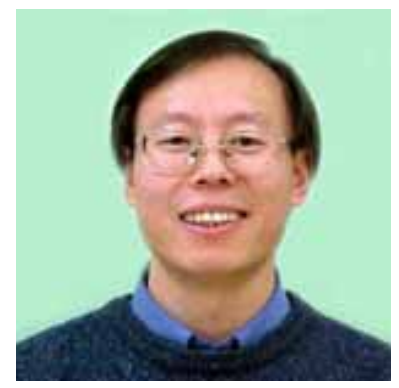

Xin-An (Lucian) Lu holds a Ph.D. in Organizational and Leadership Communication from Southern Illinois University-Carbondale. He is currently an Associate Professor in the Department of Human Communication Studies at Shippensburg University of Pennsylvania. He has published numerous articles and book chapters on leadership, organizational communication, computer-mediated communication, visual rhetoric, and women's studies. 